\title{
Social work practice in mental health: An introduction
}

\author{
Robert Bland, Noel Renouf and Ann Tullgren \\ Second edition 2015. Allen \& Unwin, NSW Australia. \\ ISBN 9781743314753 Paperback 320 pages NZD60
}

$\mathrm{T}$ This second edition of Social Work Practice in Mental Health remains an introductory text, focusing on generic practice with people with mental health problems rather than addressing key specialist areas of practice in depth. It continues to emphasise a view of social work in mental health as embracing both scientific and relational dimensions of practices that was found to be central to the successful first edition of the book published in 2005.

The book is set out in two parts. The first part explores the context for practice. In this part there is a focus on the daily-lived reality of mental illness and exploration of key concepts and issues in mental health. There is analysis and comment on the political and legal context for practice primarily in Australia. Whilst not dissimilar to the political and legal contexts in Aotearoa New Zealand, readers would be advised to locate and substitute relevant New Zealand legislation and policy to read in conjunction with this chapter. Part one concludes with a useful overview of some mental disorders, an introduction to mental health classification systems, and a description of common mental health treatments.

In the second part, focuses more on social work practice in mental health. This section begins with an orientation to assessment of mental health. The remaining chapters follow an ecological approach beginning with individual casework, moving to practice in mental health settings, mental illness service settings, working with families, and with communities. This section concludes with a discussion on sustainable social work practice in mental health agencies.

I found the authors' exploration of the practical dimensions of critically informed practice useful. There is a focus on the emergence of recovery theory as a unifying conceptual basis for mental health work, discussion of the search for evidence to inform practice, and a case made for the need to work in partnership with consumers and families in all aspects of practice.

Throughout the book the authors provide real life examples and personal narratives from people who experience mental illness which enable the reader to glimpse the daily lived reality of the impact of mental illness and the journey of recovery. Each chapter concludes with some questions for practice that prompt the reader to reflect on what they have read, and how this information can be integrated into their own practice. In addition the authors provide a list of websites providing further information and resources relevant on the each chapter topic.

The authors have captured the reality that people who experience mental health problems may engage with social work services from a variety of entry points. This book is a useful introductory text on mental health for social workers working in any field of practice. The text is easy to read, provides a comprehensive index, and useful links to further information and resources. 